\title{
Finsler-like structures from Lorentz-breaking classical particles
}

\author{
Neil Russell \\ Northern Michigan University, Marquette, MI 49855, U.S.A.
}

\begin{abstract}
A method is presented for deducing classical point-particle Lagrange functions corresponding to a class of quartic dispersion relations. Applying this to particles violating Lorentz symmetry in the minimal Standard-Model Extension leads to a variety of novel lagrangians in flat spacetime. Morphisms in these classical systems are studied that echo invariance under field redefinitions in the quantized theory. The Lagrange functions found offer new possibilities for understanding Lorentz-breaking effects by exploring parallels with Finsler-like geometries.
\end{abstract}

\section{INTRODUCTION}

In recent years, interest in the possibility of Lorentz violation in nature has blossomed. A valuable framework for the study of such deviations from the exact predictions of relativity is the Standard-Model Extension, or SME, which provides a systematic accounting of hypothesized symmetry-breaking background fields at the level of the fundamental particles in Minkowski space $[1,2]$ and in curved spacetime [3, 4]. For a recent review, see Ref. [5]. The focus of this work is on the behavior of classical particles in spacetime with Lorentz violation. This classical limit is relevant to the study of wave packets, macroscopic bodies, and relativistic scattering, among other things.

An ambitious goal would be to provide equations for classical systems moving under the effects of gravity, while interacting with each other, or with an external electromagnetic field, or with both. Significant progress towards this goal has been made in a general treatment of matter-gravity couplings in the presence of Lorentz violation [6], which includes a detailed study of the dynamics of a test body in the spin-independent limit of Lorentz violation. Based on this, atomic wave packets in gravitational free fall have been used as test bodies to investigate the Einstein equivalence principle with unprecedented precision [7], and related work has placed constraints on SME coefficients by considering atomic systems as classical bound states [8]. Alternatively, intrinsic spin can be highly amplified by atomic polarization, providing access to other combinations of Lorentz-breaking matter-gravity couplings. This has led to innovative experimental tests of Lorentz symmetry using the atomic comagnetometer and the torsion pendulum [9-11]. A recent work has explored classical motion with spin-dependent isotropic Lorentz violation [12]. Tests of fundamental physics with 
macroscopic systems in curved spacetime with Lorentz violation pose major technical challenges, and, consequently, the majority of the limits on coefficients for Lorentz violation have been attained in the flat-spacetime limit [13]. Nevertheless, searches for novel matter-gravity couplings are well justified given that some effects could be accessible only through gravitational couplings [14].

A practical approach to understanding classical behavior with Lorentz breaking is to consider, as an intial step, the flat spacetime limit in the absence of any potential. This eliminates complications due to curvature, removes the complexities of position dependence in the SME coefficients, and allows existing results from the flat-spacetime SME in field theory to be used directly. The particle trajectory follows from variation of the action $S=\int L d \lambda$, where the function $L$ is independent of position $x^{\nu}$. In the resulting equation of motion, the velocity $u^{\nu}$ is the derivative of position $x^{\nu}(\lambda)$ with respect to the trajectory parameter $\lambda$. To ensure that the physics arising from the action is independent of the parametrization, the Lagrange function $L$ must be homogeneous of degree one in the velocity $u^{\nu}$. The parameter $\lambda$ can be chosen as the proper time, but other choices may be more convenient [17]. The basic goal is then to find the classical Lagrange function $L$ for a particle of mass $m$ propagating at velocity $u^{\mu}$ in Minkowski spacetime with constant background coefficients controlling Lorentz-breaking effects. The validity of the classical limit is established by ensuring that the dispersion relation matches that of the fermion in the field theory, which is known exactly in closed form [15]. For simplicity, the present work is restricted to the minmal-SME limit, for which the eight relevant controlling coefficients are $a_{\nu}, b_{\nu}, c_{\mu \nu}, d_{\mu \nu}, e_{\nu}, f_{\nu}, g_{\lambda \mu \nu}$, and $H_{\mu \nu}$, and the dispersion relation is given by Eq. (14) of Ref. [16] or, equivalently, by Eq. (1) of Ref. [17].

A method for finding the general $L\left(u ; m, a_{\nu}, b_{\nu}, \ldots\right)$ exists [17], and is relevant for minimal and nonminimal Lorentz violation [18]. It involves finding a polynomial equation in $L$ based on the dispersion relation, the wave-packet motion, and the homogeneous form of the Lagrange function. The multiple roots $L$ reflect the spin up, spin down, matter, and antimatter states of the Dirac system. This method can be challenging to apply in the case of dispersion relations that are of higher order than quadratic in the momentum. For the special case of dispersion relations that are quadratic in the momentum, there exists a template that allows the corresponding Lagrange function to be deduced immediately [17].

To introduce the ideas and notation, a brief review of the quadratic template will be useful. Dispersion relations with quadratic dependence on the momentum $p_{\nu}$ can be put into the form

$$
p \Omega p+2 \kappa \Omega p-\mu^{2}+\kappa \Omega \kappa=0,
$$

where $\Omega^{\mu}{ }_{\nu}, \kappa_{\nu}$, and $\mu$ are constants that can be viewed as a slightly modified identity $\delta^{\mu}{ }_{\nu}$, a 
momentum shift, and a modified mass. Matrix notation is used where possible, and transposes of single-index objects are left to be inferred from the context. For example, the first term might be written $p^{\mathrm{T}} \Omega p$ in stricter matrix notation, or $p_{\sigma} \Omega^{\sigma}{ }_{\kappa} p^{\kappa}$ using indices. Raising and lowering is done with the Minkowski metric $\eta_{\mu \nu}$ with signature $(+,-,-,-)$. In the Lorentz-preserving limit, $\Omega^{\mu}{ }_{\nu} \rightarrow \delta^{\mu}{ }_{\nu}, \kappa^{\mu} \rightarrow 0$, and $\mu \rightarrow m$, so the relation becomes $p^{2}=m^{2}$. Note that $\Omega$ is always invertible, since Lorentz violation is assumed minuscule. As an example, a particle propagating in the $a_{\nu}$ and $f_{\nu}$ background has dispersion relation of this quadratic type,

$$
(p-a)^{2}-m_{a f}^{2}-(p \cdot f)^{2}=0
$$

The mass subscript takes into account the possibility that the mass enters differently for each Lorentz-breaking background. The Lagrange function corresponding to the dispersion relation (1) is

$$
L(u ; \mu, \kappa, \Omega)=-\mu \sqrt{u \Omega^{-1} u}+\kappa \cdot u .
$$

For the example corresponding to Eq. (2), $L\left(u ; m_{a f}, a, f\right)$ follows with no difficulty. Ref. [17] includes a detailed discussion of this quadratic template and further examples. Note, for example, that $L(u,-\mu, \kappa, \Omega)$ describes the antiparticle after reinterpretation.

One way to lift the Minkowski-space results into the gravitational context is to employ minimal coupling. This implies introducing dependence on position $x^{\mu}$, thereby promoting the Minkowski metric $\eta_{\mu \nu}$ to a pseudo-Riemann metric $g_{\mu \nu}(x)$, and converting the SME coefficients into functions of position. The resulting Lagrange function $L\left(x^{\mu}, u^{\mu} ; m, a_{\nu}(x), b_{\nu}(x), \ldots\right)$ depends on both position and velocity and describes a classical particle moving under the influence of gravity and subject to Lorentz breaking. The geodesics of the particle follow by applying the Euler-Lagrange equations. These ideas form the backdrop to the work presented here.

Numerous parallels exist between structures for Lorentz-breaking classical point particles in spacetime and those of Finsler and pseudo-Finsler geometries. Some of the broad literature relevant in the present context includes Refs. [19-30]. The resemblances allow results in the existing mathematical literature to be applied in some cases, suggest paths to categorizing types of classical Lorentz violation, and may provide an alternative geometrical framework that admits explicit Lorentz violation consistently [3]. Note that the signature of the underlying metric has a strong impact on the geometry. In particular, the results for Finsler manifolds, which have Riemann signature, do not necessarily carry over into pseudo-Finsler ones. However, some ties between the two signatures can be made using Wick rotation or other procedures. Recent work has highlighted the 
link between the Randers space [31] and the SME $a_{\nu}(x)$ background, identified a calculable Finsler structure based on the SME $b_{\nu}(x)$ background [32], and found others related to the $\operatorname{SME~} H_{\mu \nu}(x)$ coefficient [33]. Lagrange functions to be presented below offer avenues for additional SME-based Finsler and pseudo-Finlser structures.

There are two main sets of ideas presented. The first new result of this work is a quartic template for finding classical Lagrange functions $L\left(u ; m, a_{\nu}, b_{\nu}, \ldots\right)$ corresponding to a variety of quartic dispersion relations in flat spacetime. This extends the existing template for the case of quadratic dispersion relations discussed above, and is presented in Section II. For dispersion relations that fit the template, $L$ can be found without the contortions of the general method [17], which include eliminating momentum variables from a system of equations and factoring a highorder polynomial. Several examples involving single and multiple background fields are discussed in Section III.

The second set of results are mappings between classical systems with Lorentz violation. It is a well-established principle that physical results must be unaltered under coordinate changes and field redefinitions. Consequently, some combinations of coefficients in the SME are unobservable and can be mapped into others. In the fermion sector, literature on this topic includes Refs. [1$3,6,15,34-36]$. A first approach to the question of mappings, discussed in Section IV, is to look at isomorphisms between classical systems limited to leading order in Lorentz violation. Essentially, this translates established field-redefinition results in the quantum theory into corresponding results in the classical approach. Three particular mappings are relevant, between the $H$ and $d$ fields, between the $b$ field and the axial components of $g$, and between the $a$ and $e$ fields. The second approach asks a more challenging question, whether these three maps can be established between the exact classical systems. The finding, in section IV, is that exact many-to-one correspondences can be set up, taking the one field together with a second auxiliary SME field, onto the other. The choice of auxiliary field is not unique, and, in addition, mass redefinitions are required.

\section{CLASSICAL LAGRANGIANS FOR QUARTIC DISPERSION RELATIONS}

The new result presented here is a method for finding classical Lagrange functions corresponding to dispersion relations that are quartic in the momentum and satisfy a particular idempotent condition on the background fields.

Consider a single massive particle in Minkowski spacetime, with quartic dispersion relation

$$
\left[(p+\kappa) \Omega(p+\kappa)-\mu^{2}\right]^{2}=4(p+\kappa) S(p+\kappa),
$$


where $S^{\mu}{ }_{\nu}$, is small, constant, and vanishes in the Lorentz-preserving limit. Note that the quadratic-template dispersion relation (1) is recovered by setting $S=0$. Limiting attention to dispersion relations of this form (4) with the idempotent condition

$$
\left(S \Omega^{-1}\right)^{2}=\zeta\left(S \Omega^{-1}\right)
$$

where $\zeta$ is a real constant, the Lagrange function is

$$
L(u ; \mu, \kappa, \Omega, S)=-\left(\mu^{2}+\zeta\right)^{1 / 2} \sqrt{u \Omega^{-1} u} \mp \sqrt{u \Omega^{-1} S \Omega^{-1} u}+\kappa \cdot u .
$$

Equations (4), (5), and (6) provide a template for finding various Lagrange functions with the bipartite form [32]. The idea is to start from a given quartic dispersion relation that fits the form (4), match coefficients to get the quantities $\Omega, S, \kappa, \mu^{2}$, and arrive at the result (6) if condition (5) holds.

As an initial example, consider a fermion in an SME background controlled by the $a_{\nu}$ and $b_{\nu}$ coefficients. The dispersion relation can be written

$$
\left[(p-a)^{2}-\left(m^{2}+b^{2}\right)\right]^{2}=4(p-a)\left(b b-b^{2} \delta\right)(p-a) .
$$

Matching with Eq. (4) gives $\Omega=\delta, S^{\mu}{ }_{\nu}=b^{\mu} b_{\nu}-b^{2} \delta^{\mu}{ }_{\nu}, \kappa_{\nu}=-a_{\nu}$, and $\mu^{2}=m^{2}+b^{2}$. Condition (5) holds with $\zeta=-b^{2}$, giving

$$
L\left(u ; m_{a b}, a, b\right)=-m_{a b} \sqrt{u^{2}} \mp \sqrt{(b \cdot u)^{2}-b^{2} u^{2}}-a \cdot u,
$$

a result found earlier found using other techniques [17].

Equation (6) can be verified by extracting the canonical momentum $p_{\nu} \equiv-\partial L / \partial u^{\nu}$ from the lagrangian $L \equiv \rho+\sigma+\kappa \cdot u$ and then eliminating the velocity $u^{\nu}$ from these four equations. This amounts to imposing three conditions, and the remaining equation constraining the momenta $p_{\nu}$ is the dispersion relation (4). To give some details, note that the relationship between the canonical momentum

$$
p_{\nu}=-\frac{\mu^{2}+\zeta}{\rho}\left(\Omega^{-1} u\right)_{\nu}-\frac{1}{\sigma}\left(\Omega^{-1} S \Omega^{-1} u\right)_{\nu}-\kappa_{\nu}
$$

and the 4 -velocity $u^{\nu}$ is controlled by the quantities $\Omega$, and $S$, and $\kappa$. Evaluating $(p+\kappa) \Omega(p+\kappa)$ and $(p+\kappa) S(p+\kappa)$ with the aid of $(5)$ gives

$$
\begin{aligned}
& (p+\kappa) \Omega(p+\kappa)=2\left(\mu^{2}+\zeta\right) \frac{\sigma}{\rho}+2 \zeta+\mu^{2}, \\
& (p+\kappa) S(p+\kappa)=\left(\left(\mu^{2}+\zeta\right) \frac{\sigma}{\rho}+\zeta\right)^{2} .
\end{aligned}
$$

The velocity dependence appears as $\sigma / \rho$ in both and can be eliminated by substitution to get (4). 


\section{EXAMPLES OF CLASSICAL LAGRANGE FUNCTIONS}

\section{A. Case of restricted antisymmetric $d$}

The fermion-sector $d$ coefficient has two indices and is traceless. Denoting the symmetric part by $d_{s}$, the restriction to the antisymmetric components is made by setting $d_{s}=0$. Using this antisymmetric $d$ to establish conventions, the dual is defined by $\widetilde{d}_{\mu \nu} \equiv \frac{1}{2} \epsilon_{\mu \nu \alpha \beta} d^{\alpha \beta}$ with $\epsilon_{0123} \equiv-1$. The standard observer invariants $X$ and $Y$ for antisymmetric tensors are defined

$$
X_{d} \equiv \frac{1}{4} d_{\mu \nu} d^{\mu \nu}, \quad Y_{d} \equiv \frac{1}{4} \widetilde{d}_{\mu \nu} d^{\mu \nu},
$$

where the subscript identifies the relevant tensor.

The dispersion relation for the antisymmetric $d$ coefficient is found to be

$$
\left[p\left(\delta-d^{2}\right) p-m_{d}^{2}\right]^{2}=4 m_{d}^{2} p d^{2} p
$$

where $\left(d^{2}\right)^{\mu}{ }_{\nu}=d^{\mu}{ }_{\alpha} d^{\alpha}{ }_{\nu}$ is obtained by matrix multiplication.

Comparing coefficients with Eq. (4) gives $\kappa=0, \Omega=\delta-d^{2}, \mu^{2}=m_{d}^{2}$, and $S=m_{d}^{2} d^{2}$. The identities $d \widetilde{d}=\widetilde{d} d=-Y_{d} \delta$ and $\widetilde{d}^{2}=d^{2}+2 X_{d} \delta$ can be used to verify the antisymmetric-tensor result

$$
\left(\delta-d^{2}\right)^{-1}=\frac{\delta+\widetilde{d}^{2}}{1+2 X_{d}-Y_{d}^{2}},
$$

valid for sufficiently small $d$. Using this expression, note that $S \Omega^{-1}=m_{d}^{2}\left(d^{2}+Y_{d}^{2} \delta\right) /\left(1+2 X_{d}-Y_{d}^{2}\right)$ satisfies the projective condition (5) if the restriction $Y_{d}=0$ is made. Evaluation of $\left(S \Omega^{-1}\right)^{2}$ yields

$$
\zeta=\frac{-2 X_{d} m_{d}^{2}}{1+2 X_{d}}
$$

Substituting these results into (6), the classical Lagrange function for the restricted antisymmetric $d$ coefficient is found:

$$
L\left(u ; m_{d}, d ; d_{s}=0, Y_{d}=0\right)=-\frac{m_{d}}{1+2 X_{d}}\left[\sqrt{u^{2}+u \widetilde{d}^{2} u} \pm \sqrt{u d^{2} u}\right] .
$$

The Lagrange function for $d$, which has a bipartite structure with perturbations occurring in both square roots, has been studied in Ref. [37] using a different approach.

\section{B. Combining $b$ or $H$ with antisymmetric $c$}

The classical Lagrange function $L\left(u ; m_{b}, b\right)$ for the $b$ coefficient [32], contained in (8), has bipartite form echoed by $L\left(u ; m_{H}, H ; Y_{H}=0\right)$ for $H$ with the restriction $Y_{H}=0$ [17]. Using the 
quartic template, the Lagrange functions that combine the antisymmetric $c_{\mu \nu}$ coefficient with each of these can be deduced. In these cases, the dispersion relation takes the form

$$
\left[p\left(\delta-c^{2}\right) p-m_{c Q}^{2}+q\right]^{2}=4 p(\delta-c) Q(\delta+c) p
$$

where $Q$ is a matrix satisfying the idempotent condition $Q^{2}=q Q$. For the $b$ background, $Q=$ $b b-b^{2} \delta$ with $q=-b^{2}$, and, for the restricted $H$ background, $Q=\widetilde{H} \widetilde{H}$ with $q=2 X_{H}$. Equation (17) fits the quartic dispersion relation template (4) and matching coefficients yields $\kappa=0, \Omega=\delta-c^{2}$, $\mu^{2}=m_{c Q}^{2}-q$, and $S=(\delta-c) Q(\delta+c)$. Making use of the idempotent condition, it follows that $S \Omega^{-1}=(\delta-c) Q(\delta-c)^{-1}$ satisfies condition (5) with $\zeta=q$. The result (6) can be applied, leading to

$$
\begin{gathered}
L\left(u ; m_{b c}, b, c ; c_{s}=0\right)=-m_{b c} \sqrt{u\left(\delta-c^{2}\right)^{-1} u} \mp \sqrt{u(\delta+c)^{-1}\left(b b-b^{2} \delta\right)(\delta-c)^{-1} u}, \\
L\left(u ; m_{c H}, c, H ; c_{s}=0, Y_{H}=0\right)=-m_{c H} \sqrt{u\left(\delta-c^{2}\right)^{-1} u} \mp \sqrt{u(\delta+c)^{-1} \widetilde{H}^{2}(\delta-c)^{-1} u} .
\end{gathered}
$$

For small $c$, the inverses in these expressions exist and are found from (14) and

$$
(\delta \pm c)^{-1}=\frac{\delta \mp\left(c-Y_{c} \widetilde{c}\right)+\widetilde{c}^{2}}{1+2 X_{c}-Y_{c}^{2}} .
$$

The Lagrange function (18) for $b$ and antisymmetric $c$ describes a classical particle in a Lorentzbreaking background with ten independent degrees of freedom: four from the components of $b_{\nu}$ and six from the antisymmetric components of $c_{\mu \nu}$. In the case of (19), there are eleven independent components breaking Lorentz symmetry: six from each of the two antisymmetric tensors, with a reduction of one due to the $Y_{H}=0$ condition.

\section{Axial and Trace components of the $g$ coefficient}

Using notation from Ref. [38], the $g_{\mu \nu \lambda}$ coefficient has a unique decomposition $g_{\mu \nu \lambda}=g_{\mu \nu \lambda}^{(A)}+$ $g_{\mu \nu \lambda}^{(T)}+g_{\mu \nu \lambda}^{(M)}$ where the axial part $g_{\mu \nu \lambda}^{(A)}$ and the trace part $g_{\mu \nu \lambda}^{(T)}$ have 4 components each, and the mixed-symmetry part $g_{\mu \nu \lambda}^{(M)}$ has 16 components.

The dispersion relation for the $g$ coefficient is $[16]$

$$
0=\left(p^{2}+m_{g}^{2}-2 X_{g p}\right)^{2}-4 m_{g}^{2} p^{2}-4 p(g p)^{2} p+4 Y_{g p}^{2}
$$

where $(g p)_{\mu \nu} \equiv g_{\mu \nu \lambda} p^{\lambda} \equiv g_{\mu \nu}(p)$, and the definitions of $X$ and $Y$ follow the convention in Eq. (12), $X_{g p}=g_{\mu \nu}(p) g^{\mu \nu}(p) / 4$, and $Y_{g p}=g_{\mu \nu}(p) \widetilde{g}^{\mu \nu}(p) / 4$. 
Applying the constraint $g_{\mu \nu \lambda}^{(M)}=0$, attention is limited to cases where only the axial and trace components are nontrivial. These can be expressed in terms of 4 -vectors,

$$
A^{\alpha} \equiv g^{(A) \alpha} \equiv \frac{1}{6} g_{\sigma \kappa \tau} \epsilon^{\sigma \kappa \tau \alpha}, \quad T_{\nu} \equiv \frac{1}{3} g_{\nu}^{(T)} \equiv \frac{1}{3} g_{\nu \alpha}{ }^{\alpha}
$$

and, together with the following tensor containing information about the momentum,

$$
P_{\nu}^{\mu} \equiv p^{\mu} p_{\nu}-p^{2} \delta_{\nu}^{\mu}
$$

can be used to simplify the dispersion relation (21). It is found that $p(g p)^{2} p=p^{2} T P T$ as a result of the total antisymmetry of $g_{\mu \nu \alpha}^{(A)}$. To evaluate $X_{g p}$ and $Y_{g p}$, first note that

$$
g^{(T) \mu \nu}(p)=T^{\mu} p^{\nu}-T^{\nu} p^{\mu}, \quad g_{\mu \nu}^{(A)}(p)=\epsilon_{\mu \nu \alpha \beta} A^{\alpha} p^{\beta} .
$$

Substitution into the expression for $X_{g p}$ is aided by the identities

$$
\begin{aligned}
g_{\mu \nu}^{(A)}(p) g^{(T) \mu \nu}(p) & =0 \\
\frac{1}{2} g_{\mu \nu}^{(A)}(p) g^{(A) \mu \nu}(p) & =(A \cdot p)^{2}-A^{2} p^{2}=A P A, \\
\frac{1}{2} g_{\mu \nu}^{(T)}(p) g^{(T) \mu \nu}(p) & =-(T \cdot p)^{2}+T^{2} p^{2}=-T P T,
\end{aligned}
$$

and substitution into the expression for $Y_{g p}$ is facilitated by the identities

$$
\begin{aligned}
\frac{1}{2} g_{\mu \nu}^{(A)}(p) \widetilde{g}^{(T) \mu \nu}(p) & =(T \cdot p)(A \cdot p)-(T \cdot A) p^{2}=T P A \\
g_{\mu \nu}^{(A)}(p) \widetilde{g}^{(A) \mu \nu}(p) & =0 \\
g_{\mu \nu}^{(T)}(p) \widetilde{g}^{(T) \mu \nu}(p) & =0
\end{aligned}
$$

giving

$$
X_{g p}=\frac{1}{2} A P A-\frac{1}{2} T P T, \quad Y_{g p}=T P A .
$$

The dispersion relation (21) for the axial and trace components of $g$ takes the form

$$
0=\left(p^{2}-m_{A T}^{2}-A P A-T P T\right)^{2}-4 m_{A T}^{2} A P A+4(T P A)^{2}-4(A P A)(T P T) .
$$

The last two terms are quartic in the momentum and vanish if either $A^{\mu}=0$ or $T^{\mu}=0$. In these limits, the form matches the template (4).

To find the Lagrange function for the special case where only the trace components of $g$ are nonzero, the axial vector $A^{\mu}$ in (28) is set to zero yielding the quadratic expression $p \Omega p=\mu^{2}$, 
where ${\Omega^{\mu}}_{\nu}=\delta_{\nu}^{\mu}-T^{\mu} T_{\nu} /\left(1+T^{2}\right)$ with inverse $\left(\Omega^{-1}\right)^{\mu}{ }_{\nu}=\delta_{\nu}^{\mu}+T^{\mu} T_{\nu}$, and $\mu=m_{T} / \sqrt{1+T^{2}}$. The Lagrange function is found using the template (3) with $\kappa=0$ :

$$
L\left(u ; m_{T}, T\right)=\frac{-m_{T}}{\sqrt{1+T^{2}}} \sqrt{u^{2}+(T \cdot u)^{2}} .
$$

Note that the Finsler structure discussed in Ref. [39] has a similar structure if restricted to a single tangent space.

The Lagrange function for the $g$ coefficient with only axial components can be found by setting $T^{\mu}=0$ in (28). A match with the template dispersion relation (4) can be achieved with $\kappa=0$, $\Omega=\left(1+A^{2}\right) \delta-A A, S=m^{2}\left(A A-A^{2} \delta\right)$, and $\mu^{2}=m_{A}^{2}$. For small $A^{\mu}$, the inverse $\Omega^{-1}=$ $(\delta+A A) /\left(1+A^{2}\right)$ exists. The idempotent property (5) can be confirmed after evaluating $S \Omega^{-1}$, and it is found that $\zeta=-m_{A}^{2} A^{2} /\left(1+A^{2}\right)$. The resulting Lagrange function for axial $g$ is

$$
L\left(u ; m_{A}, A\right)=-\frac{m_{A}}{1+A^{2}}\left[\sqrt{u^{2}+(A \cdot u)^{2}} \pm \sqrt{(A \cdot u)^{2}-A^{2} u^{2}}\right] .
$$

As with Eq. (16), this Lagrange function has a bipartite form with SME coefficients in both the square roots. It is interesting to compare it with the Lagrange function $L\left(u ; m_{b}, b\right)$ contained in (8). In the quantum context, a field redefinition relates $b^{\mu}$ to $A^{\mu}$ at leading order. In the next section, this relationship is investigated in the classical-particle context. Also note that the couplings of $b^{\mu}$ or $A^{\mu}$ coefficients to fermions have the same form as matter-torsion couplings in Riemann-Cartan gravity, and this has allowed results from Lorentz tests to place tight constraints on spacetime torsion $[40,41]$.

The Lagrange functions presented in this section involve uniform Lorentz-breaking background fields in Minkowski spacetime. In this limit, classical particles undergo no acceleration, because of the homogeneity of the Lagrange function and the absence of position or parameter dependence [17]. This means constant Lorentz violation is unobservable with a single point particle. However, comparison of systems with distinct properties makes physical effects measurable. In curved spacetime, where the coefficients for Lorentz violation depend on position and time, the Lagrange functions can be viewed as Finsler-like structures. The corresponding particles follow geodesics controlled by the background fields and the curvature of the space. Geodesic equations for the $b_{\mu}(x)$ and $H_{\mu \nu}(x)$ backgrounds are known $[32,33]$, and those for others such as the ones presented in this section are left for future work. The important feature of the present Lagrange functions is that they correspond to known SME dispersion relations, so their further study in the Finsler context is of definite interest. 


\section{FIELD REDEFINITIONS AND MAPS}

In the SME, the freedom to redefine fields implies that some Lorentz-breaking fields can be mapped into others. The focus here is on the three mappings that pair the dimension-3 operators in the minimal SME with dimension-4 operators at leading order in the quantized theory. At first order in Lorentz violation, they are implemented by the replacements

$$
\begin{aligned}
\widetilde{H}_{\mu \nu} & \leftrightarrow m d_{\mu \nu}, \\
b_{\nu} & \leftrightarrow-m A_{\nu}, \\
a_{\nu} & \leftrightarrow-m e_{\nu} .
\end{aligned}
$$

In this section, each of these correspondences is verified in the case of the relevant dispersion relations and classical Lagrange functions at leading order in Lorentz violation. A second, more challenging, issue is also addressed in each case, that of whether mappings between the classical systems that hold exactly at all orders in Lorentz violation are possible. The finding is that this can be done as a many-to-one map by introducing an appropriate auxiliary background field in each case.

\section{A. Relating $H_{\mu \nu}$ to antisymmetric $d_{\mu \nu}$}

First, consider mappings between the dispersion relations at leading order in the Lorentzbreaking $H_{\mu \nu}$ and $d_{\mu \nu}$ fields. The $H$ dispersion relation for $Y_{H}=0$ can be found from Eq. (17) with appropriate substitutions for $Q$ and $q$,

$$
\left(p^{2}-m_{H}^{2}+2 X_{H}\right)^{2}=4 p \widetilde{H}^{2} p
$$

while the $d$ dispersion relation for $Y_{d}=0$ is given in Eq. (13). To express (34) at first order in Lorentz violation, take the square root of both sides, and cross out the second-order $2 X_{H}$ term. The resulting expression appears in Table I in the first entry of the second column. There is no subscript on $m$ because a mass correction can only enter at second order. Immediately below this in the table is the first-order limit of the dispersion relation (13) for $d$, which is found in a similar way. It follows by inspection that (31), which also appears in the initial column of the table, relates these first-order dispersion relations.

Next, consider mappings between the corresponding Lagrange functions at first order in Lorentz violation. Noting that $\sqrt{u^{2}+u \widetilde{d}^{2} u} \approx \sqrt{u^{2}}$ to first order in $d$, Eq. (16) becomes

$$
L\left(u ; m, d ; d_{s}=0, Y_{d}=0\right) \approx-m\left(\sqrt{u^{2}} \pm \sqrt{u d^{2} u}\right) .
$$




\begin{tabular}{|c|c|c|}
\hline Field map & First-order dispersion relations & First-order Lagrange functions \\
\hline$\widetilde{H}_{\mu \nu} \leftrightarrow m d_{\mu \nu}$ & $p^{2}-m^{2} \approx \pm 2 \sqrt{p \widetilde{H}^{2} p}$ & $L\left(u ; m, H ; Y_{H}=0\right)=-m \sqrt{u^{2}} \mp \sqrt{u \widetilde{H}^{2} u}$ \\
& $p^{2}-m^{2} \approx \pm 2 m \sqrt{p d^{2} p}$ & $L\left(u ; m, d ; d_{s}=0, Y_{d}=0\right) \approx-m\left(\sqrt{u^{2}} \pm \sqrt{u d^{2} u}\right)$ \\
\hline$b_{\nu} \leftrightarrow-m A_{\nu}$ & $p^{2}-m^{2} \approx \pm 2 \sqrt{(b \cdot p)^{2}-b^{2} p^{2}}$ & $L(u ; m, b)=-m \sqrt{u^{2}} \mp \sqrt{(b \cdot u)^{2}-b^{2} u^{2}}$ \\
& $p^{2}-m^{2} \approx \pm 2 m \sqrt{(p \cdot A)^{2}-A^{2} p^{2}}$ & $L(u ; m, A) \approx-m\left(\sqrt{u^{2}} \pm \sqrt{(A \cdot u)^{2}-A^{2} u^{2}}\right)$ \\
\hline$a_{\nu} \leftrightarrow-m e_{\nu}$ & $p^{2}-m^{2} \approx 2 a \cdot p$ & $L(u ; m, a)=-m \sqrt{u^{2}}-a \cdot u$ \\
& $p^{2}-m^{2} \approx-2 m e \cdot p$ & $L(u ; m, e) \approx-m \sqrt{u^{2}}+m e \cdot u$ \\
\hline
\end{tabular}

TABLE I: Mappings between dispersion relations and between classical Lagrange functions at first order in Lorentz-breaking background fields.

The Lagrange function for $H$ follows from Eq. (19) with $c=0$,

$$
L\left(u ; m, H ; Y_{H}=0\right)=-m \sqrt{u^{2}} \mp \sqrt{u \widetilde{H}^{2} u},
$$

and it follows by inspection that (31) relates these leading-order classical Lagrange functions, which appear in the first row and last column of Table I.

A natural progression is to seek a mapping between the full dispersion relations (34) and (13), without taking the leading-order limits. The replacement (31) is insufficient to do this. However, if the $H$ dispersion relation is augmented to include the antisymmetric $c$ coefficient as in (17) with $Q=\widetilde{H}^{2}$, an exact map to the $d$ dispersion relation (13) is indeed possible. It is implemented by the replacements

$$
\begin{aligned}
\widetilde{H}_{\mu \nu} & \rightarrow \frac{m_{d}}{\sqrt{1+2 X_{d}}} d_{\mu \nu} \\
c_{\mu \nu} & \rightarrow d_{\mu \nu} \\
m_{c H} & \rightarrow \frac{m_{d}}{\sqrt{1+2 X_{d}}} .
\end{aligned}
$$

Note that the correspondence implies $X_{H} \rightarrow-m_{d}^{2} X_{d} /\left(1+2 X_{d}\right)$. The identity $\widetilde{\widetilde{H}}=-H$ means the first expression in (37) can be written $H_{\mu \nu} \rightarrow-m_{d} \widetilde{d}_{\mu \nu} / \sqrt{1+2 X_{d}}$.

These replacements also implement an exact mapping from the classical Lagrange function $L\left(u ; m_{c H}, c, H ; c_{s}=0, Y_{H}=0\right)$ in Eq. (19) to the Lagrange function $L\left(u ; m_{d}, d ; d_{s}=0, Y_{d}=0\right)$ in Eq. (16).

This result shows that a mass redefinition is part of the morphism relating the different Lorentzbreaking systems. Although $Y_{c}$ does not need to vanish, it can be imposed without affecting the replacement $c_{\mu \nu} \rightarrow d_{\mu \nu}$, thereby reducing by one the number of independent auxiliary variables needed. With this assumption, $H_{\mu \nu}, c_{\mu \nu}$, and $d_{\mu \nu}$ each have five independent variables, and the 
map (37) can be viewed as a projection from the 11-dimensional space $\left(m_{c H}, H_{\mu \nu}, c_{\mu \nu}\right)$ to the 6-dimensional space $\left(m_{d}, d_{\mu \nu}\right)$. Since the mapping is many-to-one, it is not invertible.

\section{B. Relating $b_{\nu}$ to $g_{\nu}^{(A)}$}

To investigate the classical version of the mapping (32), the first-order dispersion relations for $b$ and $A$ are needed. The expression for $b$ is found by setting $a=0$ in (7) and keeping only the first order terms in $b$. The result appears in Table I, row two, column two. Immediately below it is the dispersion relation for the $A$ background, obtained from the exact result (28) with $T=0$,

$$
0=\left(p^{2}-m_{A}^{2}-A P A\right)^{2}-4 m_{A}^{2} A P A,
$$

by taking the first-order limit. Inspection of the entries in the table shows that (32) maps the two leading-order dispersion relations.

To verify the mapping (32) at the level of the classical Lagrange functions, note the following. The Lagrange function (8) with $a=0$ involves a correction to the conventional Lorentz-preserving Lagrange function that is already first order in Lorentz violation $b$, and appears in Table I, row two, column three. The first-order approximation to the $A_{\nu}$ Lagrange function (30),

$$
L(u ; m, A) \approx-m\left(\sqrt{u^{2}} \pm \sqrt{(A \cdot u)^{2}-A^{2} u^{2}}\right)
$$

appears below it in the table. By inspection of the table entries, it can readily be seen that the replacement (32) establishes an isomorphism between the $b_{\nu}$ and $A_{\nu}$ Lagrange functions at first order.

With the use of an additional SME background field, $f_{\mu}$, an exact mapping can be established between the all-orders dispersion relation for $b$ and $f$,

$$
0=\left(p^{2}-(f \cdot p)^{2}-m_{b f}^{2}-b^{2}\right)^{2}-4 b P b,
$$

and that of $A$, given in Eq. (38). The map is defined by the replacements

$$
\begin{aligned}
b_{\nu} & \rightarrow \frac{m_{A} A_{\nu}}{1+A^{2}}, \\
f_{\nu} & \rightarrow \frac{A_{\nu}}{\sqrt{1+A^{2}}}, \\
m_{b f} & \rightarrow \frac{m_{A}}{1+A^{2}} .
\end{aligned}
$$

As with the morphism in (37), this map involves a mass redefinition and an auxiliary field. Note that the auxiliary $f_{\nu}$ has the same number of independent components as $b_{\nu}$ and $A_{\nu}$. This property 
is also true for the $c_{\mu \nu}$ auxiliary field used in mapping from $H_{\mu \nu}$ to $d_{\mu \nu}$. The morphism (41) is many-to-one, taking the 9-dimensional space $\left(m_{b f}, b_{\nu}, f_{\nu}\right)$ to the 5 -dimensional space $\left(m_{A}, A_{\nu}\right)$.

\section{Relating $a_{\nu}$ to $e_{\nu}$}

The dispersion relations for a particle in the $a$ background or in the $e$ background are

$$
\begin{aligned}
(p-a)^{2}-m_{a}^{2} & =0, \\
p^{2}-\left(m_{e}-e \cdot p\right)^{2} & =0 .
\end{aligned}
$$

The first-order approximations given in row three, column two of Table I, follow by dropping the subscripts on the masses and keeping only terms that are linear in Lorentz violation. It follows by inspection of these entries in the table that the replacement (33) implements the map between the two leading-order dispersion relations.

Since the dispersion relations (42) and (43) are quadratic in the momentum, the corresponding classical Lagrange functions follow by application of the quadratic template, and are known to be

$$
\begin{aligned}
L\left(u ; m_{a}, a\right) & =-m_{a} \sqrt{u^{2}}-a \cdot u, \\
L\left(u ; m_{e}, e\right) & =-\frac{m_{e}}{1-e^{2}}\left\{\sqrt{u^{2}+(e \cdot u)^{2}-e^{2} u^{2}}-e \cdot u\right\} .
\end{aligned}
$$

Table I contains $L(u ; m, a)$ in row three, column three since it is already first-order in Lorentz violation. Immediately below it is the first-order approximation to $L(u ; m, e)$. Inspection of these entries shows that the replacement (33) maps the leading-order Lagrange functions into each other, as it does in the quantum case.

Next, consider the question of finding an exact mapping between the $a$ and $e$ systems, linking the dispersion relations (42) and (43), and the Lagrange functions (44) and (45). As seen in the earlier examples involving the maps (37) and (41), this can be done with the assistance of an auxiliary field. The auxiliary field is not unique, and to confirm this, exact mappings are given using two different auxiliary fields.

A first mapping to the full $e$ dispersion relation (43) may be established by using $T_{\nu}$ as the auxiliary field. To find the dispersion relation for the combined $a$ and $T$ coefficients, the steps leading to $(28)$ can be repeated with a few modifications, including the assumption $A_{\nu}=0$. The result is

$$
0=\left((p-a)^{2}-m_{a T}^{2}-T P T\right)^{2}+4 p M p
$$


where $M_{\nu}^{\mu}$ is defined by $M=\left((a \cdot T)^{2}-a^{2} T^{2}\right) \delta+a^{2} T T-(a \cdot T)(a T+T a)+T^{2} a a$. An exact map from this dispersion relation for $a$ and $T$ to that for $e$, Eq. (43), is found to be

$$
\begin{aligned}
a^{\mu} & \rightarrow \frac{-m_{e} e^{\mu}}{1-e^{2}}, \\
T^{\mu} & \rightarrow \frac{e^{\mu}}{\sqrt{1-e^{2}}}, \\
m_{a T} & \rightarrow \frac{m_{e}}{1-e^{2}} .
\end{aligned}
$$

To verify that this map works at the level of the classical Lagrange functions, $L\left(u ; m_{a T}, a, T\right)$ is needed. While the dispersion relation (46) is quartic in the momentum, it does not appear to fit the quartic template in Sec. II, and this Lagrange function is unknown at present.

Having seen that $T$ can serve as an auxiliary field to implement an exact map from the $a$ system to the $e$ system, the next point to be made is that this auxiliary is not unique. To demonstrate this, the $f_{\nu}$ field is used as an alternative auxiliary to implement the $a$ to $e$ mapping. The dispersion relation for the combined $a$ and $f$ coefficients is given in Eq. (2), and an exact mapping to (43) is made using the replacements

$$
\begin{aligned}
a_{\nu} & \rightarrow-m_{e} e_{\nu}, \\
f_{\nu} & \rightarrow e_{\nu} \\
m_{a f} & \rightarrow \sqrt{1+e^{2}} m_{e} .
\end{aligned}
$$

To show that these replacements also map the Lagrange functions, $L\left(u ; m_{a f}, a, f\right)$ is needed. Since the corresponding dispersion relation (2) is quadratic, the template (3) can be applied and the result is

$$
L\left(u ; m_{a f}, a, f\right)=-\sqrt{m_{a f}^{2}+\frac{(a \cdot f)^{2}}{1-f^{2}}} \sqrt{u^{2}+\frac{(f \cdot u)^{2}}{1-f^{2}}}-a \cdot u-\frac{a \cdot f}{1-f^{2}} f \cdot u .
$$

It can be verified that the substitutions (48) implement a map from (49) to (45).

Note the properties seen in the (37) and (41) morphisms are echoed in both cases here. Exact mappings from $a_{\nu}$ to $e_{\nu}$ can be constructed with the aid of an auxiliary field with the same number of components as $a$ and $e$. In (47) this is the $T_{\nu}$ field, and in (48) it is the $f_{\nu}$ field, demonstrating that the auxiliary is not unique. The map also involves a mass redefinition. It is many-to-one, and takes 9 variables consisting of a mass, the four components $a_{\nu}$, and the four components of the auxiliary field, to the 5 variables $\left(m_{e}, e_{\nu}\right)$. 


\section{SUMMARY AND DISCUSSION}

The main result presented in section II is a classical Lagrange function in Minkowski space that describes a massive particle in the context of minimal Lorentz violation with certain quartic dispersion relations. The result is given in the form of a template: any particle with dispersion relation matching (4), and with Lorentz-breaking background satisfying the idempotent condition (5), has Lagrange function given in equation (6).

The quartic template is used to provide explicit Lagrange functions for several minimal-SME Lorentz-breaking backgrounds that have not appeared in the literature to date. The results for the functions $L\left(u ; m_{b c}, b, c, c_{s}=0 ; c_{s}=0\right)$, and $L\left(u ; m_{c H}, c, H ; c_{s}=0, Y_{H}=0\right)$, show that Lorentz violation matching the quartic template and combining more than one background field can be studied with relative ease. Earlier work [17] provided a template for Lagrange functions with quadratic dispersion relations and included the explicit form of $L\left(u ; m_{a e f}, a, e, f\right)$, which has twelve independent components for the background fields. The quartic examples in this work provide explicit Lagrange functions in (18) and (19) with ten and eleven independent background-field components respectively. The Lagrange function $L\left(u ; m_{T}, T\right)$ involving the four trace components of the SME $g$ background, denoted $T_{\nu}$, is given in equation (29). In the case of Lorentz violation by the axial components $A_{\nu}$ of the $g$ background, $L\left(u ; m_{A}, A\right)$ appears in equation (30). Finding the classical Lagrange function for all the 24 independent components of the $g$ background field remains an open challenge.

In section IV, mappings between SME background fields have been studied. The leadingorder isomorphisms between $\widetilde{H}$ and $d$, between $b$ and axial $g$, and between $a$ and $e$ are known in the fundamental field-theoretic formalism to arise because of the freedom to redefine fields and coordinates. The same leading-order correspondences are confirmed in the classical limit, and summarized in Table I.

The second aspect studied in section IV is exact mappings between the above minimal-SME background fields. Isomorphisms are not expected, because each SME field at the level of the fundamental Lagrange function controls a different field operator. It is found that exact projective mappings do exist, in each case taking more than one SME field onto another SME field. For the three leading-order mappings already discussed, exact projective mappings are established with the aid of a second SME background field, which at least doubles the number of independent field components in the domain space. A feature of these projective maps is a rescaling of the mass that depends on the fields. This means the mass can be considered an independent component on 
the same footing as the field components. For the $H$ to $d$ map, the antisymmetric components of the $c$ background provide an auxiliary field, so that the domain $\left(m_{H c}, H_{\mu \nu}, c_{\mu \nu}\right)$ with $Y_{H}=0$ has dimension 12 and maps to the range $\left(m_{d}, d_{\mu \nu}\right)$ with $Y_{d}=0$, of dimension 6 . For the $b_{\nu}$ to $A_{\nu}$ map, the field $f_{\nu}$ can be used as an auxiliary and the projection takes the 9-dimensional domain $\left(m_{b f}, b_{\nu}, f_{\nu}\right)$ onto the 5-dimensional range $\left(m_{A}, A_{\nu}\right)$. The auxiliary field used in these projective mappings is not unique, as can be seen in the case of the $a$ to $e$ map, for which $f_{\nu}$ is one possible auxiliary and $T_{\nu}$, the trace component of $g$, is another. This gives a projection from the 9-dimensional domain space $\left(m_{a f}, a_{\nu}, f_{\nu}\right)$ or $\left(m_{a T}, a_{\nu}, T_{\nu}\right)$ onto the 5 -dimensional range space $\left(m_{e}, e_{\nu}\right)$.

The quartic template in Eqs. (4), (5), and (6) is applicable in a limited set of cases and a natural question is how to generalize it. Staying within the minimal SME, one direction for further investigation would be to seek ways to weaken the idempotent condition (5). This would be useful, for example, in addressing the Lagrange functions $L\left(u ; m_{b f}, b, f\right)$ and $L\left(u ; m_{a T}, a, T\right)$, where the respective dispersion relations, (40) and (46), match (4), but the background fields do not satisfy the idempotent condition (5). Another goal in the minimal SME context would be to broaden the template to more general quadratic dispersion relations, covering for example equation (28), the case of $g_{\lambda \mu \nu}$ limited to axial and trace components. The most ambitious goal in the minimal SME would be to find the Lagrange function corresponding to the full dispersion relation appearing in Ref. [16]. This is likely to be a very long and unenlightening expression, given that even the case of $L\left(u ; m_{H}, H\right)$ for a general $H$ background with nonzero $Y_{H}$ is known to be highly complex [17]. There are numerous options for further development. Other investigations might include methods for finding Lagrange functions for nonminimal Lorentz violation [15], or for interacting particles.

The main motivation of this work is to provide new Finsler-like structures that are rooted in the Lorentz-breaking background fields of the minimal SME. The new Minkowski-space classical Lagrange functions found here can be used as the basis for Finsler and pseudo-Finsler structures in future work. They hold the promise of providing insights into the geometrical nature of Lorentz violation in classical systems and of gaining new insights into the physical content based on existing approaches in Finsler geometry. 


\section{Acknowledgments}

Thanks to the Indiana University Center for Spacetime Symmetries for hospitality while this research was undertaken.

[1] D. Colladay and V.A. Kostelecký, Phys. Rev. D 55, 6760 (1997) [hep-ph/9703464].

[2] D. Colladay and V.A. Kostelecký, Phys. Rev. D 58, 116002 (1998) [hep-ph/9809521].

[3] V.A. Kostelecký, Phys. Rev. D 69, 105009 (2004) [hep-th/0312310].

[4] Q.G. Bailey and V.A. Kostelecký, Phys. Rev. D 74, 045001 (2006) [gr-qc/0603030].

[5] J.D. Tasson, Rep. Prog. Phys. 77, 062901 (2014) [arXiv:1403.7785].

[6] V.A. Kostelecký and J.D. Tasson, Phys. Rev. D 83, 016013 (2011) [arXiv:1006.4106].

[7] M.A. Hohensee, S. Chu, A. Peters, and H. Müller, Phys. Rev. Lett. 106, 151102 (2011) [arXiv:1102.4362].

[8] M.A. Hohensee, H. Müller, and R.B. Wiringa, Phys. Rev. Lett. 111151102 (2013) [arXiv:1308.2936].

[9] J.D. Tasson, Phys. Rev. D 86, 124021 (2012) [arXiv:1211.4850].

[10] J.M. Brown, S.J. Smullin, T.W. Kornack, and M.V. Romalis, Phys. Rev. Lett. 105, 151604 (2010) [arXiv:1006.5425].

[11] H. Panjwani, L. Carbone, and C.C. Speake, in CPT and Lorentz Symmetry V, edited by V.A. Kostelecký, World Scientific, Singapore, 2011.

[12] M. Schreck, arXiv:1409.1539.

[13] V.A. Kostelecký and N. Russell, Rev. Mod. Phys. 83, 11 (2011) [2014 edition arXiv:0801.0287v7].

[14] V.A. Kostelecký and J.D. Tasson, Phys. Rev. Lett. 102, 010402 (2009) [arXiv:0810.1459].

[15] V.A. Kostelecký and M. Mewes, Phys. Rev. D 88, 096006 (2013) [arXiv:1308.4973].

[16] V.A. Kostelecký and R. Lehnert, Phys. Rev. D 63, 065008 (2001) [arXiv:hep-th/0012060].

[17] V.A. Kostelecký and N. Russell, Phys. Lett. B 693, 443 (2010) [arXiv:1008.5062].

[18] M. Schreck, arXiv:1405.5518.

[19] P. Finsler, Über Kurven und Flächen in allgemeinen Räumen, University of Göttingen dissertation, 1918; Verlag Birkhäuser, Basel, Switzerland, 1951.

[20] D. Bao, S.-S. Chern, and Z. Shen, An Introduction to Riemann-Finsler Geometry, Springer, New York, 2000.

[21] Z. Shen, Canad. J. Math. 55, 112 (2003).

[22] D. Bao, C. Robles, and Z. Shen, J. Diff. Geom. 66, 377 (2004).

[23] G.Yu. Bogoslovsky, Phys. Lett. A 350, 5 (2006) [hep-th/0511151].

[24] C. Pfeifer and M.N.R. Wohlfarth, Phys. Rev. D 84, 044039 (2011) [arXiv:1104.1079].

[25] S.I. Vacaru, Class. Quant. Grav. 28, 215001 (2011) [arXiv:1008.4912]. 
[26] C. Lämmerzahl, V. Perlick, and W. Hasse, Phys. Rev. D 86, 104042 (2012) [arXiv:1208.0619].

[27] M.A. Javaloyes and M. Sánchez, Int. J. Geom. Methods Mod. Phys. 111460032 (2014) [arXiv:1311.4770].

[28] J.E.G. Silva and C.A.S. Almeida, Phys. Lett. B 731, 74 (2014) [arXiv:1312.7369].

[29] J.M. Romero, O. Sánchez-Santos, and J.D. Vergara, Phys. Lett. A 375, 3817 (2011) [arXiv:1106.3529].

[30] A.P. Kouretsis, Eur. Phys. J. C 74, 2879 (2014) [arXiv:1312.4631].

[31] G. Randers, Phys. Rev. 59, 195 (1941).

[32] V.A. Kostelecký, Phys. Lett. B 701, 137 (2011) [arXiv:1104.5488].

[33] V.A. Kostelecký, N. Russell, and R. Tso, Phys. Lett. B 716, 470 (2012) [arXiv:1209.0750].

[34] B. Altschul, J. Phys. A 39, 13757 (2006) [hep-th/0602235].

[35] R. Lehnert, Phys. Rev. D 74, 125001 (2006) [hep-th/0609162].

[36] D. Colladay and P. McDonald, J. Math. Phys. 43, 3554 (2002) [hep-ph/0202066].

[37] D. Colladay and P. McDonald, Phys. Rev. D 85, 044042 (2012) [arXiv:1201.3931].

[38] A. Fittante and N. Russell, J. Phys. G 39, 125004 (2012) [arXiv:1210.2003].

[39] R.G. Beil, Int. J. Theor. Phys. 28, 659 (1989).

[40] V.A. Kostelecký, N. Russell, and J.D. Tasson, Phys. Rev. Lett. 100, 111102 (2008) [arXiv:0712.4393].

[41] R. Lehnert, W.M. Snow, and H. Yan, Phys. Lett. B 730, 353 (2014) [arXiv:1311.0467]. 